\title{
APROPIACIÓN TECNOLÓGICA EN EL MANEJO DE COMPETENCIAS DIGITALES POR LOS PROFESORES DE ESCUELAS NORMALES EN MÉXICO
}

\section{TECHNOLOGICAL APPROPRIATION IN THE MANAGEMENT OF DIGITAL COMPETENCES BY TEACHERS OF NORMAL SCHOOLS IN MEXICO}

\section{Arnoldo Lizárraga Juárez ${ }^{1}$}

\section{Evangelina López Ramírez²}

\section{Jorge Eduardo Martínez Iñiguez ${ }^{3}$}

Universidad Autónoma De Baja California

Baja California, México

1 UNIVERSIDAD AUTÓNOMA DE BAJA CALIFORNIA BAJA CALIFORNIA, MÉXICO. M.C. Arnoldo Lizárraga. Maestro en Educación y doctorante del programa Innovación en Tecnología Educativa de la Universidad Autónoma de Querétaro. Profesor en la Licenciatura en Ciencias de la Educación. Correo arnoldo.lizarraga@uabc.edu.mx https://orcid.org/0000-0003-42287823

2 UNIVERSIDAD AUTÓNOMA DE BAJA CALIFORNIA BAJA CALIFORNIA, MÉXICO. Dra. Evangelina López. Editor Académico de la Revista "Espíritu Científico en Acción" de la Secretaría de Educación en Baja California. Evaluadora de programas universitarios, proyectos de investigación y publicaciones científicas y revistas indizadas. Profesor/investigador en la Facultad de Ciencias Humanas UABC. Forma parte del Cuerpo Académico "Formación, desarrollo y evaluación de actores educativos. Miembro del Sistema nacional de investigadores (SNI México). Correo evangelinalopez@uabc.edu.mx https:// orcid.org/0000-0003-0960-1788

3 UNIVERSIDAD AUTÓNOMA DE BAJA CALIFORNIA BAJA CALIFORNIA, MÉXICO. Dr. Eduardo Martínez. Doctorado en Socioformación. Profesor-investigador de la Facultad de Ciencias Humanas de la Universidad Autónoma de Baja California, México. Conferencista e instructor de cursos en países como Colombia, México y el Salvador. Sus líneas de investigación versan sobre calidad de la educación, currículo, educación superior y socioformación. Es miembro de la Sociedad Mexicana de Educación Comparada. Miembro del Sistema nacional de investigadores (SNI México).Correo jorge.martinez43@uabc.edu.mx https://orcid.org/0000-0002-8833-5600 


\section{RESUMEN}

El propósito de este documento es referir los resultados del estudio realizado con profesores de Escuelas Normales (formadoras de docentes) de México relativo a los niveles de apropiación que estos manejan sobre las Tecnologías de la información y la comunicación como apoyo a su práctica docente. Las evidencias establecen que este nivel fue bajo, lo que indica una limitada capacidad para diseñar e implementar escenarios educativos apoyados en las tecnologías cuestión que restringe la participación activa del alumno, su comprensión profunda de los contenidos de aprendizaje, ni su actividad constructiva. Esta condición representa un espacio de oportunidad significativo para el futuro próximo ya que estos recursos se vuelven cada vez más indispensables en el quehacer educativo.

\section{PALABRAS CLAVE}

Profesores, apropiación de las tecnologías de la información y comunicación

\section{ABSTRACT}

The purpose of this document is to refer the results of the study carried out with teachers of Normal Schools (teacher educators) of Mexico regarding the levels of appropriation that they handle on Information and communication technologies in support of their teaching practice. The evidence establishes that this level was low, which indicates a limited capacity to design and implement educational scenarios supported by the technologies that restrict the active participation of the student, their deep understanding of the learning content, and their constructive activity. This condition represents a significant opportunity space for the near future as these resources become increasingly indispensable in the educational endeavor.

\section{KEY WORDS}

Teachers, appropriation of information and communication technologies

\section{INTRODUCCIÓN}

El ritmo acelerado en que las Tecnologías de la Información y la Comunicación (TIC) han incursionado en la vida del ser humano, también ha transformado la manera en que éste se desempeña e interactúa en todos los contextos, desde lo cotidiano hasta lo profesional (Magro et al., 2014). A través de recursos como las computadoras, el internet y los dispositivos móviles, el mundo del siglo XXI está experimentando un acceso sin precedentes a la información, un desvanecimiento de barreras geográficas, la capacidad de comunicarse de forma instantánea y simultánea y la difusión y multiplicación de las opiniones (Flores, Galicia y Sánchez, citado en Fandiño, 2011).

Con este panorama en mente, se han llevado a cabo numerosas reflexiones sobre el impacto, posibilidades y retos que ha traído consigo la incursión de la tecnología digitalen los contextos sociales y educativos, así como el papel que desempeña la escuela en la actualidad y su necesidad de renovarse en todas sus áreas para atender a las demandas de las generaciones contemporáneas (Hernández, Gamboa y Ayala, 2014; Valencia et al., 2016). Esto no es asunto tangencial sobre todo para la educación superior, puesto que la formación profesional del individuo para su integración efectiva en la sociedad actual, así como su actuación pertinente en un campo laboral cada vez más complejo, supone inevitablemente la actualización de las Instituciones de Educación Superior (IES) en todas sus funciones $y$ dimensiones, incluyendo la curricular y la docente. Por ello la insistencia de reforzar la formación de los profesores en el manejo de las TIC para inducir un ejercicio óptimo, moderno 
y pertinente en un contexto donde los cambios aparecen continuamente(Cabero, Marín y Castaño, 2015; Valencia et al., 2016).

Es así que el Gobierno mexicano, a través de la Secretaría de Educación Pública (SEP), ha derivado acciones hacia las Escuelas Normales situando diversos programas y reformas que han buscado integrar el uso de las TICen las aulas desde hace más de tres décadas, tanto en los planes y programas de estudio, como en apoyo a los procesos educativos que se llevan a cabo en ellas (Gutiérrez, 2015). Actualmentela tecnología digital cuenta con una presencia significativa en la educación normal gracias ala reformulación de los Planes de Estudio 2012, de los cuales han llegado a formar parte a través de su inclusión en diversas asignaturas y en las competencias del perfil de egreso (Gutiérrez, 2015).

De acuerdo con Acosta, Martínez y Perea (2015), en la actualidadexisten pocas investigaciones que describan o expliquen las maneras en que los docentes de educación normal incorporan lastecnologías dentro de sus prácticas. Sin embargo, según autores como Rodríguez (2014), Gutiérrez (2015) y Palmares (2015), a pesar de los avances en materia de infraestructura y capacitación en las Escuelas Normales, los docentes de dichas instituciones continúan atribuyéndoles un uso limitado.

\section{PROBLEMA Y OBJETIVO DE LA INVESTIGACIÓN}

Considerando factores como la presencia generalizada de las TIC en el mundo del siglo $\mathrm{XXI}$, la transición hacia un modelo educativo marcado por las demandas de una sociedad informatizada y la inclusión formal de las tecnologíasinformáticas en el currículo de las instituciones formadoras de docentes del país, los educadores de las escuelas normales se encuentran ante la necesidad de desarrollar nuevas competencias y habilidades que les permitan fortalecer su práctica, particularmente en su dimensión pedagógica y didáctica (Valencia et al., 2016; Zempoalteca, Barragán, Martínez y Flores, 2017).

Por lo anterior, resulta necesario realizar estudios que generen información relevante sobre el estado actual de los profesores de las Escuelas Normales de B.C. en cuanto al uso de las TIC en apoyo a su práctica docente.Partiendo de esta idea, el propósito de este escrito es presentar los resultados de una investigación realizada en dichas instituciones, basada en la propuesta de los niveles de apropiación de las tecnologías informáticas, también denominada apropiación tecnológica.

El problema fue: ¿Cuál es el nivel de apropiación tecnológica en el manejo de competencias digitales por parte de los docentes de las Escuelas Normales de B.C.?El objetivo principal del trabajo fue analizar dicho nivel de apropiación, con el fin de identificar las áreas que requieren ser fortalecidas para el manejo de las tecnologías desde la dimensión pedagógica. La difusión de los resultados de esta investigación será buen punto de partida para diseñar acciones encaminadas a mejorar la práctica docente y en esa medida contribuir a elevar la calidad de la educación ofrecida en estas instituciones.

\section{NECESIDADES DE FORMACIÓN DOCENTEEN EL SIGLO XXI:}

\section{COMPETENCIAS DIGITALES PEDAGÓGICAS}

Desde hace años se ha señalado la disconformidad que existe con la educación que ofrecen los centros educativos y con la formación profesional que reciben los docentes en México y el resto de América Latina (Braslavsky, 1999). Sobre esto, Braslavksy (1999) expone que el fortalecimiento de la competencia pedagógico- 
didáctica es un aspecto importante en el proceso de resignificación y reinvención de la práctica docente, acciones necesarias para hacer frente a los diversos desafíos a los que se enfrenta la profesión desde hace décadas. De acuerdo a esta autora, la transformación de esta dimensión implica contemplar la inclusióndel uso de las TIC, tanto por los mismos docentes como por los alumnos.

La formación y capacitación de los docentes en el empleo de tecnologías de la información y la comunicación, así como en su adecuada integración en los procesos educativos, implica el desarrollo de nuevas habilidades y competencias vinculadas a la alfabetización informacional, al dominio técnico de herramientas digitales y a la articulación de dichos conocimientos con saberes pedagógicos y disciplinarios (Rangel, 2015; Flores y Roig, 2016).Hernández, Gamboa y Ayala (2014) enfatizan que las competencias docentes en TIC, enmarcadas en la capacidad de diseñar experiencias de aprendizaje significativas donde los estudiantes adopten el papel central, son necesarias para hacer frente a los retos de la sociedad del siglo XXI.

Desde esa perspectiva, el término competencia digital docente ha sido utilizado en la literatura para referirse a la movilización y puesta en acción de todos aquellosconocimientos, habilidades, destrezas y consideraciones éticas que permitan el uso reflexivo y creativo de las TIC en el abordaje satisfactorio de las situaciones que puedan surgir en todos los ámbitos de desempeño de un docente, incluyendo el pedagógico (Hernández, Gamboa y Ayala, 2014; Flores y Roig, 2016). Este concepto, desde la dimensión pedagógica, hace referencia a aquellas competencias que permiten el uso intencional de las TIC como apoyo a los procesos de enseñanza y aprendizaje y en cuya movilización intervienen tanto conocimientos y habilidades comunicativos e instrumentales, como de planificación, desarrollo y evaluación de la acción formativa (Hernández, Gamboa y Ayala, 2014).

Para Cabero y Marín (2014), un punto de referencia inicial en el tema son las políticas que se han formulado en torno a los estándares de formación publicados por diferentes organismos e instituciones a nivel nacional e internacional. En el caso del estándarque orienta el presente estudio, "Competencias y Estándares TIC Desde la Dimensión Pedagógica", publicado por UNESCO y la Pontificia Universidad Javeriana - Cali(Valencia et al., 2016), se priorizan tres tipos de competencias en el uso educativo de las TIC que, según esta propuesta, deben fundamentar los planes de formación docente relativos a la apropiación de las tecnologías: las de diseño, implementación y evaluación de escenarios educativos mediados por TIC. Estos escenarios pueden ser comprendidos como las situaciones de aprendizaje que se manifiestan en una lección o curso, a través de la interacción de elementos como los actores del proceso educativo, los objetivos de aprendizaje, los contenidos, los métodos, las actividades, los recursos y la evaluación (Valencia et al., 2016).

Lacompetencia de diseñarescenarioseducativos apoyados en TIC refiere los conocimientos y habilidades de planificación y organización de todos aquellos elementos que permitan la construcción de escenarios educativos donde intervenga el uso de las tecnologías y se promueva el aprendizaje significativo del estudiante.La de implementar escenarios educativos apoyados en TIC se relaciona con las habilidades que permiten la puesta en acción del diseño de un escenario educativo particular, mientras que la de evaluar la efectividad de los escenarios educativos apoyados en TIC implica las habilidades que permiten al docente valorar la efectividad de los escenarios educativos para favorecer el aprendizaje significativo de los estudiantes (Valencia et al., 2016). 
La puesta en acción de estas tres competencias implica que el docente sea capaz de identificar recursos TIC de diversos tipos, cómo se utilizan y sus usos potenciales como herramientas mediadoras en los procesos de enseñanza y aprendizaje. Además, conlleva la toma de consciencia sobre los cambios que ocurren en los procesos de aprendizaje cuando se diseñan, seleccionan y utilizan recursos y actividades donde intervienen las tecnologías (Valencia et al., 2016).

Como exponen Hernández, Acevedo, Martínez y Cruz (2014), las TIC son instrumentos que pueden fungir como recursos pedagógicos, pero la orientación que se le da a su uso depende, en gran parte, de la capacidad y habilidades de los docentes y alumnos y de las interacciones que estos actores tengan con las tecnologías en el aula. En ese sentido, la concepción que los docentes tienen sobre las TIC y sus usos potenciales en el diseño, implementación y evaluación de escenarios educativos se ve directamente impactada por el nivel en el que se ubica su apropiación de las tecnologías al incorporarlas en sus prácticas pedagógicas, incidiendo de diversas maneras en las situaciones de aprendizaje y en sus resultados (Valencia et al., 2016).

\section{NIVELES DE APROPIACIÓN TECNOLÓGICA}

Según Herodotou, Winters y Kambouri (citado en Del Valle, Celaya y Ramírez, 2016), la apropiación tecnológica, o apropiación de las TIC, puede ser definida como la adopción, adaptación e incorporación de las tecnologías por parte de los usuarios en sus prácticas cotidianas. Partiendo de esta idea, se comprende la apropiación tecnológica en el ámbito de la docencia como "la manera en que los docentes incorporan las TIC a sus actividades cotidianas de clase" (Valencia et al., 2016: 11). Esta apropiación no ocurre súbitamente, sino que se desarrolla a lo largo de diversas fases que autores como Montes y Ochoa (2006) y Valencia et al. (2016) han denominado niveles de apropiación. Estos mismos autores proponen tres niveles: integración, reorientación y evolución.

Cuando el uso de las TIC en las prácticas pedagógicas se ubica en el nivel de integración, la concepción que se tiene de ellas es como herramientas que facilitan la búsqueda, el almacenamiento, la transmisión y el intercambio de información de forma efectiva (Prince y Figueroa, 2015; Valencia etal., 2016). Al momento de diseñar, implementar y evaluar los escenarios educativos, se aprovechan los beneficios de las TIC como recursos que permiten agilizar $y$ hacer más efectivos determinados procesos que en la mayoría de los casos podrían realizarse sin el uso de la tecnología, pero de una manera más lenta y menos eficiente. Para Valencia et al. (2016), las decisiones respecto al uso de recursos tecnológicos se ven supeditadas a las novedades que presentan "con relación a la economía de tiempo, dinero y versatilidad" ( $p$. 19).

Debido a que en este nivel el uso de las TIC en escenarios educativos se centra en mejorar la gestión cotidiana de los mismos, las tecnologías se utilizan principalmente para la exposición de los temas de la clase; el envío o publicación de anuncios e instrucciones a los alumnos sobre actividades a realizar; la búsqueda, gestión y digitalización de documentos y, en general, la flexibilización del manejo de tiempo, espacio y recursos. Asimismo, se utilizan y promueve el uso las TIC para acceder, buscar y recolectar información de calidad relacionada con los contenidos de la asignatura(Montes y Ochoa, 2006; Valencia et al., 2016).

Por otra parte, cuando los usos pedagógicos de las TIC se ubican en el nivel de reorientación, éstas dejan de ser utilizadas principalmente como herramientas que permiten poner a disposición 
de los estudiantes grandes cantidades de información de forma rápida, fácil y económica. En su lugar, pasan a ser concebidas como recursos que promueven la participación activa y la construcción de conocimiento por parte de los alumnos, a partir de sus características particulares: interactividad, dinamismo, multimedia, hipermedia y conectividad (Valencia et al., 2016).

Según Valencia et al. (2016), "gracias a la optimización y aprovechamiento de esas características, las TIC pueden ser utilizadas por estudiantes y docentes para planificar, regular y orientar los procesos intra e intermentales implicados en la construcción de conocimiento" (p. 20). Estos rasgos son los que permiten acceder, utilizar y transformar la información en diferentes formas de representación, posibilitando, entre otras cosas, la simulación y modelamiento de problemas y su solución.

Por último, el nivel de evolución implica que el docente tiene claro el potencial sin precedentes que tienen las TIC para integrar los sistemas semióticos conocidos y ampliar la capacidad humana en la representación, procesamiento, transmisión y compartición de información (Coll, Mauri y Onrubia, 2008; Valencia et al., 2016). Además, el docente utiliza las TIC para mediar las interacciones entre él y otros docentes, instituciones y grupos de investigación (Valencia et al., 2016). Aunado a esto, dada la claridad que tiene el docente en este nivel, colabora con colegas en el diseño de escenarios educativos apoyados con las tecnologías y se preocupa por divulgar sus conocimientos y experiencias en materia de los usos pedagógicos de las TIC (Valencia et al., 2016).

También debe destacarse que en este último nivel el docente se preocupa por mantenerse actualizado y buscar información sobre los últimos avances $y$ tendencias educativas mediadas por la tecnología. Además, realiza innovaciones en sus clases apoyadas con TIC, buscando desarrollar y proponer nuevos empleos para las herramientas tecnológicas(Valencia et al., 2016).

\section{METODOLOGÍA}

\section{Tipo de estudio}

Se realizó un estudio no experimental con enfoque cuantitativo y de alcance descriptivo. De acuerdo a Hernández, Fernández y Baptista (2010), los diseños de investigación no experimentales se caracterizan porque el investigador no manipula deliberadamente las variables, sino que se observan los fenómenos tal como ocurren en su contexto natural, para posteriormente analizarlos. Por su parte, los estudios de alcance descriptivo tienen como propósito describir la realidad investigativa sin explicar causas, especificando las características, propiedades y perfiles de las personas, objetos, o fenómenos que sean sometidos a análisis (Hernández, Fernández y Baptista, 2010). Se optó por este enfoque debido a que no se busca valorar el grado de asociación entre variables, ni establecer relaciones causales, sino analizar y especificar las características de un determinado número de sujetos de investigación en relación al uso que otorgan a las TIC en su labor como docentes.

La muestra se conformó por 151 docentes de escuelas normales de sostenimiento público en el estado de Baja California, con edades que oscilan entre los 30 a 59 años de edad y con una experiencia de entre 1 a 19 años de servicio. De acuerdo a información del Sistema de Información Básica de la Educación Normal (SIBEN), el total de la población de docentes que laboran en las diez escuelas normales públicas del estado es de 250 (SIBEN, 2017). En cuanto al cálculo de la muestra, se utilizó la fórmula , lo que determinó el número de participantes requeridos con un nivel de confianza del $95 \%$. 
El instrumento utilizado para recolectar la información fue un cuestionario de 21 ítems, validado por 4 expertos en materia de diseño y contenido. En cuanto a las variables que se consideraron, el instrumento evaluódiversos aspectos relacionados con el nivel de apropiación tecnológica de los docentes en el manejo de las competencias de diseño, implementación y evaluación de escenarios educativos apoyados en TIC, así como los niveles de capacitación en que se perciben los docentes en cuanto al uso de las tecnologías (ver tabla 1).
Para definir los niveles de apropiación tecnológica en el manejo de cada competencia digital se tomó como base principal y se adaptó el estándar diseñado por Valencia et al. (2016), publicado por la Pontificia Universidad Javeriana - Cali con el apoyo de la Oficina de Santiago de UNESCO. Asimismo, se utilizaron como referencia los trabajos de Montes y Ochoa (2006) y Prince y Figueroa (2015) en la definición de los indicadores para cada uno de los niveles de apropiación.

Tabla 1

Variables que evalúa el instrumento

\begin{tabular}{|c|c|}
\hline Variables & $\begin{array}{c}\text { Número de ítems } \\
\text { por variable }\end{array}$ \\
\hline Capacitación en el uso de las TIC por parte de los docentes. & 3 \\
\hline Diseño de escenarios educativos apoyados en TIC. & 6 \\
\hline Acciones en apoyo a los procesos de enseñanza y aprendizaje \\
utilizando las TIC. & 2 \\
\hline Evaluación de los aprendizajes utilizando las TIC & 3 \\
\hline Evaluación de escenarios educativos apoyados en TIC. & 4 \\
\hline Comunicación de conocimientos relacionados con el diseño, \\
implementación y evaluación de escenarios educativos apoyados en \\
TIC.
\end{tabular}

Fuente: elaboración propia.

\section{ANÁLISIS DE LA INFORMACIÓN}

Para analizar los datos se llevó a cabo un análisis estadístico descriptivo utilizando el programa SPSS. La interpretación de los datos se enfocó en identificar la relación entre la información ofrecida por los participantes y los niveles de apropiación de las tecnologías informáticas relativos a las tres competencias consideradas en el estudio: diseño, implementación y evaluación de escenarios educativos apoyados con tecnologías informáticas. Cabe señalar que la mayoría de los ítems ofrece información particular a cada una de las competencias consideradas en el estudio, con la excepción del ítem relativo a los niveles de capacitación de los docentes en el uso de tecnologías informáticas, puesto que la identificación y comprensión del funcionamiento de dichas herramientas se consideró información relevante tanto para la competencia de diseño, como de implementación de escenarios educativos apoyados en la tecnología. 


\section{RESULTADOS}

El primer dato que resalta es que lastecnologías informáticasen cuyo uso los docentes se perciben como más capacitados son principalmente las que permiten la búsqueda, almacenamiento, comunicación, transmisióne intercambio de información de manera efectiva, es decir las asociadas con el nivel de integración. Entre las opciones que mostraron mayores porcentajes en la categoría de "capacitado o muy capacitado" se encuentran los motores de búsqueda en línea (86.1\%), equipo de cómputo y sus periféricos (84.1\%), equipo para proyectar presentaciones y video $(82.8 \%)$ y software para comunicar e intercambiar información escrita (81.5\%). Por el contrario, la mayoría señaló considerarse "poco o nada capacitado" en el uso de recursos que permiten explotar las características particulares de las TIC y favorecer la construcción de conocimiento, como lo son las herramientas de la web 2.0 y las que permiten interactuar con la información de formas novedosas y constructivas.

Con relación a la competencia de diseño de escenarios educativos apoyados en TIC, se encontró que un $74.1 \%$ de docentes la pone en acción con regularidad, sin embargo al hacerlo priorizan aspectos relacionados principalmente con el nivel de integración. Sobre esto, los datos indican que al planear y organizar sus clases apoyadas con TIC, los criterios que la mayoría de lossujetospriorizan son su efectividad para presentar los contenidos de la asignatura $(80 \%)$, sus beneficios en el acceso y búsqueda de información de calidad relacionada con los contenidos de la asignatura (70.7\%) y su efectividad para comunicar y transmitir información (69.3\%).Asimismo, resalta el hecho de que sólo un $38 \%$ prioriza la participación activa del alumno al momento de planear sus clases y actividades apoyadas en dichos recursos.
Respecto al manejo de la competencia de implementación de escenarios educativos apoyados en TIC, se encontró que su nivel de apropiación es el de integración. Esto se sustenta en el hecho de que las actividades que los docentes manifestaronrealizar "casi siempre o siempre" en sus asignaturas con el apoyo de las TIC son principalmente aquellas donde las tecnologías facilitan la búsqueda, presentación y comunicación de información. En este rubro, las opciones que mostraron porcentajes mayoritarios son la búsqueda de información relacionada con los contenidos de la asignatura por parte del docente $(83.3 \%)$ y por parte del alumno (82.6\%), la exposición de temas utilizando proyector y diapositivas por parte del alumno (81.4\%) y el docente (76\%), y el intercambio de información utilizando correo electrónico u otros medios digitales (72\%).En contraste, las actividades menos realizadas con apoyo de las TIC son las que permitirían niveles más avanzados de mediación entre los alumnos y los contenidos de aprendizaje, las que favorecen su actividad constructiva y las que fomentan su interacción y colaboración.

Una de las características de los niveles de apropiación avanzados en el manejo de la competencia anterior es el aprovechamiento del potencial de las TIC para evaluar y dar seguimiento a los procesos constructivos de los estudiantes. Sin embargo, se encontró que un $62.3 \%$ no realiza esta actividad con regularidad con el apoyo de las TIC, por lo que se considera que no están conscientes de dicho potencial, o no lo aprovechan del todo. En cuanto a los usos principales que les otorgan en este proceso, las opciones que mostraron porcentajes mayoritarios nuevamente se vinculan directamente con el nivel de integración, mostrándose en el rango de entre el $67.9 \%$ al $75.6 \%$, mientras que las opciones de niveles superiores, como lo son el dar seguimiento a los niveles de participación de los alumnos, o retroalimentar a los mismos en relación a su desempeño y progreso durante 
la asignatura, se mantienen entre el $22.1 \%$ y el $37.4 \%$

En relación a la evaluación de la efectividad de los escenarios educativos apoyados en TIC, se encontró que un porcentaje del $74.9 \%$ no maneja esta competencia, o no lo hace con una frecuencia suficiente, y al hacerlo no acostumbra utilizar herramientas tecnológicas. Es importante destacar que todos los niveles de apropiación considerados en el estudio suponen el uso de las TIC en la evaluación de dichos escenarios, por lo que analizando en conjunto los datos anteriores, no se puede afirmar que los docentes alcanzan el nivel más bajo de apropiación en el manejo de esta competencia.

Además de los datos anteriores, se debe señalar que los docentes no comparten con regularidad sus conocimientos relacionados con los usos educativos de las TIC en ninguna de las competencias evaluadas, mostrando porcentajes por debajo del $40 \%$ en la categoría de "casi siempre o siempre".

\section{CONCLUSIONES}

El dominio y manejo de las tecnologías informáticas por parte de los profesores normalistas para una incorporación pedagógica significativa en los procesos formativos se ha convertido en un desafío para las instituciones educativas y para quienes son responsables de programas, proyectos o estudios que tienen como referente estas nuevas condiciones emergentes. En el caso particular de las escuelas normales, autores como Rodríguez (2014) y Gutiérrez (2015) han señalado los retos a los que éstas se enfrentan en este rubro, subrayando que el uso que sus docentes otorgan a las diversas tecnologías en materia pedagógica ha sido limitado y poco relevante.

Lo anterior coincide con los resultados de esta investigación, en la que se pone de manifiesto que las Escuelas Normales del estado de Baja California se enfrentan al desafío de transformar las maneras en que las TIC son incorporadas a las prácticas cotidianas de enseñanza de sus formadores de docentes, con el fin de que éstas contribuyan a generar experiencias de aprendizaje significativas y pertinentes.Esto se sustenta en el hallazgo de que, al diseñar e implementar escenarios educativos apoyados en las tecnologías, el nivel de apropiación tecnológica de los profesores normalistas es el más bajo, es decir el de integración, lo que no favorece la participación activa del alumno, su comprensión profunda de los contenidos de aprendizaje, ni su actividad constructiva. Aunado a lo anterior, los docentesde dichas instituciones no cuentan con una cultura de la evaluación de su propia práctica en lo referente al uso de las TIC, puesto que sólo un porcentaje significativamente minoritario del $25.1 \%$ señaló movilizar esta competencia con regularidad.

Los resultados del estudio ponen de manifiesto la necesidad de llevar a cabo acciones que contribuyan a elevar el nivel de apropiación tecnológica del personal docente de las Escuelas Normales del estado. Un primer acercamiento podría ser el de abordar los problemas de capacitación percibidos por los mismos sujetos de investigación, quienes manifiestan sentirse poco o nada capacitados en el uso de recursos de la web 2.0 y otros relacionados con niveles de apropiación superiores para apoyar el aprendizaje de los alumnos. Asimismo, es importante señalar que cualquier esfuerzo de formación o actualización docente en el manejo de las tecnologías debe enmarcarse en una visión socioconstructivista de su empleo, asumir diversos niveles de capacitación y contemplar diferentes dimensiones de sus usos relacionados con la práctica pedagógica, como lo son la integración dinámica del conocimiento tecnológico, el pedagógico y el del contenido disciplinar (Cabero, 2014; Cabero y Marín, 2014). 


\section{REFERENCIAS BIBLIOGRÁFICAS}

Braslavsky, C. (1999). Bases, orientaciones y criterios para el diseño de programas de formación de profesores. Revista Iberoamericana de Educación, 19, 1350. Recuperado de: https://rieoei.org/ historico/oeivirt/ rie19a01.htm

Cabero, J. (2014). Formación del profesorado universitario en TIC. Aplicación del Método Delphi para la selección de los contenidos formativos. Educación $X X I, 17$ (1), 109-132. doi: 10.5944/ educxx1.17.1.10707

Cabero, J. y Marín, V. (2014). Miradas sobre la formación del profesorado en tecnologías de información y comunicación (TIC). Enl@ce: Revista Venezolana de Información, Tecnología y Conocimiento, 11 (2), 11-24. Recuperado de: https://dialnet.unirioja. es/descarga/articulo/5101939.pdf

Cabero, J., Marín, V. y Castaño, C. (2015). Validación de la aplicación del modelo TPACK para la formación del profesorado en TIC. @TIC. Revista d'Innovació Educativa, 14, 13-22. doi: 10.7203/attic. 14.4001

Coll, C., Mauri, T. y Onrubia, J. (2008). La utilización de las tecnologías de la información y la comunicación en la educación: Del diseño tecno-pedagógico a las prácticas de uso. En C. Coll \& C. Monereo (Eds.), Psicología de la educación virtual. Aprender y enseñar con las Tecnologías de la Información y la Comunicación (pp. 74-103). Madrid: Morata Ediciones.

Del Valle, D., Celaya, R. y Ramírez, M. S. (2016). Apropiación tecnológica en el movimiento educativo abierto: Un estudio de casos de prácticas educativas abiertas. Revista Iberoamericana de Educación, 70 (1), 149-166. Recuperado de: http:// www.rieoei.org/deloslectores/7186.pdf

Fandiño, Y. J. (2011). La educación universitaria en el siglo XXI: de la sociedad de la información a la sociedad del conocimiento. Revista Iberoamericana de Educación, Boletín 55/3. Recuperado de: https://rieoei.org/historico/ jano/3965Fandino_Jano.pdf

Flores, C. y Roig, R. (2016). Diseño y validación de una escala de autoevaluación de competencias digitales para estudiantes de pedagogía. Pixel-Bit. Revista de Medios y Educación, 48, 209-224. DOI: http://dx.doi.org/10.12795/pixelbit.2016. i48.14

Gutiérrez, J. H. (2015). Las Tecnologías de la Información y la Comunicación (TIC) y la transformación de las Escuelas Normales de México. Ponencia presentada en el XVI Congreso Virtual Educa México 2015, en el Seminario de Formación Docente "Formación, evaluación y certificación docente". Recuperado de: http://www.virtualeduca. red/ documentos/23/150420\%20 Ponencia\%20Propuesta $\% 20-\% 20$ Jorge \% 20 Gutierrez \% 20 - \% 20 VirtualEduca\%202015.pdf

Hernández, C. A., Gamboa, A. A. y Ayala, E. T. (2014). Competencias TIC para los docentes de educación superior. Ponencia presentada en Congreso Iberoamericano de Ciencia, Tecnología, Innovación y Educación, Buenos Aires, Argentina. Recuperado de: http://www. oei.es/congreso2014/memoriactei/837. pdf 
Hernández, L., Acevedo, J. A., Martínez, C. \& Cruz, B. C. (2014). El uso de las TIC en el aula: un análisis en términos de efectividad $y$ eficacia. Ponencia presentada en Congreso Iberoamericano de Ciencia, Tecnología, Innovación y Educación, Buenos Aires, Argentina. Recuperado de: www.oei. es/historico/congreso2014/memoriactei 1523.pdf

Hernández, R., Fernández, C. \& Baptista M. P. (2010). Metodología de la investigación(Quinta edición). México: McGraw-Hill.

Magro, C., Salvatella, J., Álvarez, M., Herrero, O., Paredes, A. y Vélez, G. (2014). 8 competencias digitales para el éxito profesional. Barcelona, España: Roca Salvatella. Recuperado de:http://www. rocasalvatella.com/sites/default/files/ maqueta_competencias_espanol.pdf

Montes, J. A. \& Ochoa, S. (2006). Apropiación de las tecnologías de la información y comunicación en cursos universitarios. Acta Colombiana de Psicología, 9 (2), 87-100. Recuperado de: http://www. redalyc.org/pdf/798/79890209.pdf

Palmares, O. G. (2015). Uso de las TIC por parte de los docentes de la Escuela Normal de Educación Preescolar. Ponencia presentada en $\mathrm{V}$ Congreso Nacional e Internacional de Estudios Comparados en Educación. Recuperado de: http:// www.saece.org.ar /docs/congreso5/ trab049.pdf

Prince, M. S. y Figueroa, M. (2015. Exploración de la apropiación tecnológica en profesores de universidades latinoamericanas que incorporan Recursos Educativos Abiertos (REA) en clases presenciales. Revista de Investigación Educativa de la Escuela de Graduados en Educación, 6 (11), 36-43. Recuperado de: http:// rieege.tecvirtual.mx/index. php/rieege/ article/view/141

Rangel, A. (2015). Competencias docentes digitales: propuesta de un perfil. PixelBit. Revista de Medios y Educación, 46, 235-248. doi: http://dx.doi.org/10.12795/ pixelbit.2015.i46.15

Rodríguez, M. (2014). Elconocimiento yactitud de los docentes de las Escuelas Normales ante el uso de las TIC. Recuperado de: http://registromodeloeducativo. sep.gob.mx/ Archivo;jsessionid=b92 93ef1f15189c78a58931fc9e4?nomb re $=2505-e l+$ conocimiento+y +actitud+ + de+los+docentes+de+las+escuelas+ normales+ante+el+uso+de+las+TIC.pdf

SIBEN. (2017). Difusión BD Docentes Octubre 2016. Recuperado el mes de mayo de 2017 de http://www.siben.sep.gob.mx/

Valencia, T., Serna, A., Ochoa, S., Caicedo, A. M., Montes, J. A. y Chávez, J. D. (2016). Competencias y estándares TIC desde la dimensión pedagógica: Una perspectiva desde los niveles de apropiación de las TIC en la práctica educativa docente. Cali: Pontificia Universidad Javeriana; UNESCO. Recuperado de: http://www.unesco.org/new/ fileadmin/ MULTIMEDIA/FIELD/Santiago/pdf/ Competencias-estandares-TIC.pdf

Zempoalteca, B., Barragán, J. F., González, J. y Guzmán, T. (2017). Formación en TIC y competencia digital en la docencia en instituciones públicas de educación superior. Apertura, 9 (1), 80-96. DOI: http://dx.doi.org/10.18381/Ap.v9n1.922 